\title{
ON PROGNOSIS OF VARIATION OF PROPERTIES OF EPITAXIAL LAYERS BY CHOOSING OF TEMPERATURE FIELD DURING GROWTH
}

\author{
Evgeny L. Pankratov* \\ Nizhny Novgorod State University, 23 Gagarin avenue, Nizhny Novgorod, 603950, Russia \\ Nizhny Novgorod State Technical University, 24 Minin Street, Nizhny Novgorod, 603950, Russia \\ *Corresponding author: elp2004@mail.ru
}

\begin{abstract}
In this paper we analyze influence of the temperature of growth of epitaxial layers during this technological process. Several conditions to increase homogeneity of the considered epitaxial layers were formulated. An analytical approach for analysis of mass and heat transfer was introduced. The approach gives a possibility to take into account changes of parameters of processes simulta-neously in space and simultaneously in time. At the same time the approach gives a possibility to take into account nonlinearity of the considered processes.
\end{abstract}

Key words: Epitaxial growth, Properties of epitaxial layers, Homogeneity of properties.

\section{INTRODUCTION}

Development of solid-state electronics and a wide application of heterostructures for manufacturing of electronic devices leads to the need for an improvement of properties of the materials used. For the manufacturing of heterostructures one can use different methods such as molecular beam epitaxy, epitaxy from the gas phase, magnetron sputtering. A large quantity of works about manufacturing and experimental studies of hetero-structures were published. For example, Stepanenko (1980), Gusev and Gusev (1991) and Lachin and Savelov (2001) describe main classical ideas of epitaxy processes. Vorob'ev et al. (2003), Sorokin et al. (2008), Lundin et al. (2009), Li et al.(2006), Chakraborty et al. (2004) and Mitsuhara et al. (1998) describe different developments of epitaxy to growth of specific optoelectronic and power applications. At the same time smaller number of papers, see for example (Talalaev et al., 2001), is devoted to predicting epitaxy processes. In the present paper a two-layer structure has been consider. This structure consists of a substrate and a growing epitaxial layer (see figure 1). The main aim of the paper is an analysis of changes of the temperature during growth on properties of the layer. An accompanying aim of the paper is development of analytical approach for analyzing of mass and heat transfer, which simultaneously takes into account changes in the parameters of processes both in space and in time, as well as the nonlinearity of these processes.

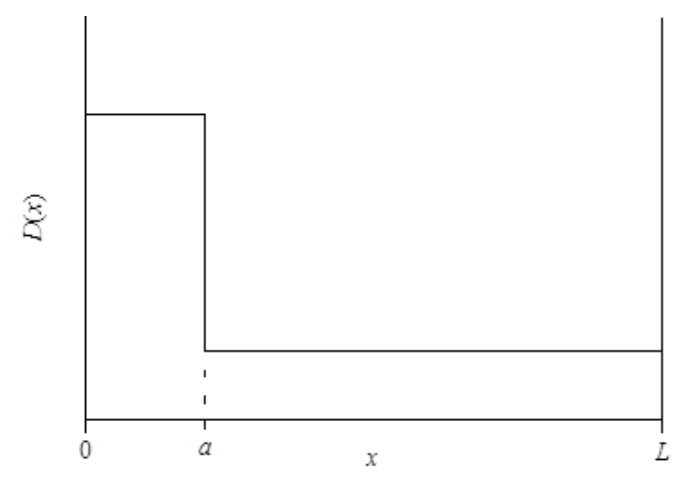

Fig. 1. Heterostructure, which consist of a substrate and an epitaxial layer. 


\section{METHOD OF THE SOLUTION}

To solve aim of the present paper concentration of the growth component $\mathrm{C}(\mathrm{x}, \mathrm{t})$ has been considered. The required concentration has been determined by solving of the following boundary value problem:

$$
\begin{aligned}
& \frac{\partial C(x, t)}{\partial t}=\frac{\partial}{\partial x}\left[D_{C} \frac{\partial C(x, t)}{\partial x}\right] \\
& \left.\frac{\partial C(x, t)}{\partial x}\right|_{x=-v t}=\left.0 \quad \frac{\partial C(x, t)}{\partial x}\right|_{x=L}=0 \\
& C(x, 0)=f(x)
\end{aligned}
$$

It should be noted that the material of the epitaxial layer does not reach the boundary $x=L$. For this reason, we can assume that $C(L, t)=0$. In relations (1) and (2), the following notation is introduced: $T$ is the temperature of growth, $D_{C}$ is the coefficient of mutual diffusion, $v$ is the speed of epitaxial growth. Value of the coefficient of mutual diffusion depends on properties of materials of layers of the multilayer structure, rate of heating and cooling of the multilayer structure. Dependences of the diffusion coefficient on the parameters could be approximated by the following relation, see for example (Gotra, 1991):

$$
D_{C}=D_{L}(x, T)\left[1+\xi \frac{C^{\gamma}(x, t)}{P^{\gamma}(x, T)}\right]
$$

Here $D_{L}(x, T)$ is the dependence of the considered diffusion coefficient on coordinate $x$ (due to dependence of properties of the heterostructure on coordinate) and temperature; $P(x, T)$ is the limit of solubility of material of epitaxial layer (if we consider only epitaxial layer than $\xi=0$ ); determined by the properties of the material, parameter $\gamma$ could be integer with values in the following interval $\gamma \in[1,3]$, see for example (Gotra, 1991). The concentration dependence of the diffusion coefficient wss discussed in detail in (Gotra, 1991). Next, we replace variables $\mathrm{x}$ and $\mathrm{t}$ and the dif-fusion coefficient $D_{L}(x)$ to the dimensionless variables $\chi$ and $\vartheta$ and the normalized diffusion coefficient $\Delta(x)$ introduced by the following relations $x \rightarrow \chi=(x+v t) / a, t \rightarrow v=t D_{0} / a^{2}$ and $\Delta$ $=D_{L}(x) / D_{0}$, where $D_{0}$ is the average value of the diffusion coefficient. Using the replacement leads to transformation of diffusion equation (1) to the following form:

$$
\frac{\partial C(\chi, \vartheta)}{\partial \vartheta}=
$$$$
\frac{\partial}{\partial \chi}\left\{\Delta(\chi, \vartheta)\left[1+\varsigma \frac{C^{\gamma}(\chi, \vartheta)}{P^{\gamma}(\chi, \vartheta)}\right] \frac{\partial C(\chi, \vartheta)}{\partial \chi}\right\}
$$

$-\mu \frac{\partial C(\chi, \vartheta)}{\partial \chi}$

Here $\mu=v a / D_{0}$. Boundary and initial conditions take the new form: $C(x+\Xi, 0)=f(\chi), \mathrm{J}(0, \vartheta)=0$, $C(X+\Xi, \vartheta)=0$, where $X=L / a, \Xi=v a \vartheta / D_{0}$. Since near the boundary $\mathrm{x}=L$ (i.e., $\chi=X+\Xi$ ) of the multilayer structure considered in figure 1 , the term $\Xi$ can be neglected in comparison with the term $X$. The approximation gives a possibility to write the last boundary condition in a simplified form: $\mathrm{C}(X, \vartheta)=$ 0 and to analyze mixing of materials of the multilayer structure in a moving region with the length $X$. To estimate the spatio-temporal distribution of material of epitaxial layer, analytical approaches attracted higher interest in comparison with numerical ones, due to greater visibility of solution as a function of physical and technological parameters. The analytical estimation of the function $C(\chi, \vartheta)$ could be done by solving equation (4). Next the equation is transformed to the equivalent integro-differential form.

$$
\begin{aligned}
C(\chi, \vartheta)= & \int_{0}^{\chi} \frac{1}{\Delta(v, \vartheta)} \int_{0}^{v} \frac{\partial C(u, \vartheta)}{\partial \vartheta} d u d v \\
& +\int_{0}^{\chi} \frac{\mu}{\Delta(v, \vartheta)} \int_{0}^{v} \frac{\partial C(u, \vartheta)}{\partial u} d u d v \\
& -\varsigma \int_{0}^{\chi} \frac{C^{\gamma}(v, \vartheta)}{P^{\gamma}(v, \vartheta)} \frac{\partial C(v, \vartheta)}{\partial v} d v
\end{aligned}
$$

Solution of equation (5) is obtained by using of method of averaging functional corrections, see for example (Sokolov, 1955). To obtain the first-order approximation of the considered concentration by using the above method the function $C(\chi, \vartheta)$ and its derivatives should be replaced by their average values on the right side of equation (3): $C(\chi, \vartheta) \rightarrow \alpha_{1}$, $\partial C(\chi, \vartheta) / \partial \vartheta \rightarrow \beta_{1}$ and $\partial C(\chi, \vartheta) / \partial \chi \rightarrow \omega_{1}$. Further the required solution has been calculated. However, to increase speed of convergence of the method of averaging functional corrections and to calculate function $C(\chi, \vartheta)$ with higher accuracy, more accurate zero-order approximation in the right side of equation (5) has to be used. As a more accurate zeroorder approximation of function $C(\chi, \vartheta)$ we use the solution of the diffusion equation in the simplest 
case: with averaged diffusion coefficient and fixed in time boundaries of the structure of the epitaxial layer - substrate. It can be shown, see for example (Pankratov, 2004), that averaged diffusion coefficient $D_{0}$ is usually the most appropriate zero-order approximation of changes in the diffusion coefficient in the case, when these changes are not very large. The solution of the diffusion equation with averaged diffusion coefficient could be obtained by standard methods, see for example (Tikhonov \&
Samarskii, 1972; Carslaw \& Jaeger, 1964), and written in the following form:

$$
\tilde{C}(\chi, \vartheta)=2 \sum_{n=0}^{\infty}\left\{\begin{array}{c}
f(\chi) \cos [\pi(n+0.5) \chi] d \chi \\
\times \cos [\pi(n+0.5) \chi] \\
\times \exp \left[-\pi^{2}(n+0.5)^{2} \vartheta\right]
\end{array}\right.
$$

Substituting of relation (6) into equation (5) gives a possibility to obtain the first-order approximation of the concentration of the epitaxial layer material in the substrate:

$$
\begin{aligned}
& C_{1}(\chi, \vartheta)=\pi \varsigma \sum_{n=0}^{\infty} \exp \left[-\pi^{2}(n+0.5)^{2} \vartheta\right] \int_{0}^{\mathrm{x}} f(\chi) \cos [\pi(n+0.5) \chi] d \chi \int_{0}^{\chi} \frac{\sin [\pi(n+0.5) v]}{\Delta(v, \vartheta) P^{\gamma}(v, \vartheta)} \\
& \times\left[\sum_{m=0}^{\infty} \int_{0}^{\mathrm{x}} f(\chi) \cos [\pi(m+0.5) \chi] d \chi \cos [\pi(m+0.5) \chi] \exp \left[-\pi^{2}(m+0.5)^{2} \vartheta\right]\right]^{\gamma} d v(n+0.5) \\
& \times 2^{\gamma+1}-2 \pi \sum_{n=0}^{\infty} \int_{0}^{\mathrm{x}} f(\chi) \cos [\pi(n+0.5) \chi] d \chi \exp \left[-\pi^{2}(n+0.5)^{2} \vartheta\right] \int_{0}^{\chi} \frac{\sin [\pi(n+0.5) v]}{\Delta(v, \vartheta)} d v \\
& \times(n+0.5)+2 \mu \sum_{n=0}^{\infty} \int_{0}^{\chi} \Delta^{-1}(v, \vartheta) \cos [\pi(n+0.5) v] d v \int_{0}^{\mathrm{x}} f(\chi) \cos [\pi(n+0.5) \chi] d \chi \\
& \times \exp \left[-\pi^{2}(n+0.5)^{2} \vartheta\right]
\end{aligned}
$$

The second-order approximation of the function $C(\chi, \vartheta)$ can be obtained by the standard replacement (Sokolov, 1995), i.e. $C(\chi, \vartheta) \rightarrow \alpha_{2}+C_{1}(\chi, \vartheta)$. The on the right side of equation (4), see for example

$$
C_{2}(\chi, \vartheta)=\int_{0}^{\chi} \frac{1}{\Delta(\chi, \vartheta)} \int_{0}^{v} \frac{\partial C_{1}(u, \vartheta)}{\partial \vartheta} d u d v+\mu \int_{0}^{\chi} \frac{C_{1}(v, \vartheta)}{\Delta(v, \vartheta)} d v-\varsigma \int_{0}^{\chi}\left[\frac{\alpha_{2}+C_{1}(v, \vartheta)}{P(v, \vartheta)}\right]^{\gamma} \frac{\partial C_{1}(v, \vartheta)}{\partial v} d v
$$

Parameter $\alpha_{2}$ could be determine by the following relation (Sokolov, 1955):

$\alpha_{2}=\frac{1}{\Theta \mathrm{X}} \int_{0}^{\Theta x} \int_{0}^{\mathrm{X}}\left[C_{2}(\chi, \vartheta)-C_{1}(\chi, \vartheta)\right] d \chi d \vartheta$

Substitution of relations (7) and (8) into (9) leads to the following result:

$$
\begin{aligned}
\alpha_{2}(\gamma=1) & =\left[\varsigma \int_{0}^{\Theta} \int_{0}^{\Theta x} \chi \frac{C_{1}(\chi, \vartheta)}{P(\chi, \vartheta)} \frac{\partial C_{1}(\chi, \vartheta)}{\partial \chi} d \chi d \vartheta-\int_{0}^{\Theta} \int_{0}^{x} \int_{0}^{\chi} \frac{\partial C_{1}(u, \vartheta)}{\partial \vartheta} d u \frac{\chi d \chi d \vartheta}{\Delta(\chi, \vartheta)}\right. \\
& \left.-\int_{0}^{\Theta x} \int_{0}^{\mathrm{X}} C_{1}(\chi, \vartheta) d \chi d \vartheta-\mu \int_{0}^{\Theta x} \int_{0} C_{1}(\chi, \vartheta) \frac{\chi d \chi}{\Delta(\chi, \vartheta)}\right]\left[\Theta \mathrm{X}-\varsigma \int_{0}^{\Theta} \int_{0}^{\mathrm{X}} \chi \frac{C_{1}(\chi, \vartheta)}{P(\chi, \vartheta)} \frac{\partial C_{1}(\chi, \vartheta)}{\partial \chi} d \chi d \vartheta\right]^{-1}
\end{aligned}
$$




$$
\begin{aligned}
& \alpha_{2}(\gamma=2)=-\frac{2 \varsigma \int_{0}^{\Theta} \int_{0}^{\mathrm{X}} \chi \frac{C_{1}(\chi, \vartheta)}{P^{2}(\chi, \vartheta)} \frac{\partial C_{1}(\chi, \vartheta)}{\partial \chi} d \chi d \vartheta-\Theta \mathrm{X}}{2 \varsigma \int_{0}^{\Theta} \int_{0}^{\Theta} \chi \frac{C_{1}(\chi, \vartheta)}{P^{2}(\chi, \vartheta)} \frac{\partial C_{1}(\chi, \vartheta)}{\partial \chi} d \chi d \vartheta} \\
& +\left\{\frac{2 \varsigma \int_{0}^{\Theta x} \int_{0}^{\mathrm{X}} \chi \frac{C_{1}(\chi, \vartheta)}{P^{2}(\chi, \vartheta)} \frac{\partial C_{1}(\chi, \vartheta)}{\partial \chi} d \chi d \vartheta-\Theta \mathrm{X}}{2 \varsigma \int_{0}^{\Theta x} \frac{\partial C_{1}(\chi, \vartheta)}{\partial \chi} \frac{\chi d \chi d \vartheta}{P^{2}(\chi, \vartheta)}}-\frac{4}{\varsigma \int_{0}^{\Theta} \int_{0}^{\mathrm{X}} \frac{\partial C_{1}(\chi, \vartheta)}{\partial \chi} \frac{\chi d \chi d \vartheta}{P^{2}(\chi, \vartheta)}}\right. \\
& \times\left[\varsigma \int_{0}^{\Theta} \int_{0}^{\mathrm{X}} \chi \frac{C_{1}^{2}(\chi, \vartheta)}{P^{2}(\chi, \vartheta)} \frac{\partial C_{1}(\chi, \vartheta)}{\partial \chi} d \chi d \vartheta-\int_{0}^{\Theta} \int_{0}^{\mathrm{X}} \frac{\chi}{\Delta(\chi, \vartheta)} \int_{0}^{\chi} \frac{\partial C_{1}(u, \vartheta)}{\partial \vartheta} d u d \chi d \vartheta\right. \\
& \left.\left.-\mu \int_{0}^{\Theta} \int_{0}^{\mathrm{x}} \chi \frac{C_{1}(\chi, \vartheta)}{\Delta(\chi, \vartheta)} d \chi d \vartheta-\int_{0}^{\Theta} \int_{0}^{\Theta x} C_{1}(\chi, \vartheta) d \chi d \vartheta\right]^{2}\right\}^{\frac{1}{2}} \\
& \alpha_{2}(\gamma=3)=\sqrt[3]{\sqrt{\frac{p^{3}}{27}+\frac{q^{2}}{4}}-\frac{q}{2}}-\sqrt[3]{\sqrt{\frac{p^{3}}{27}+\frac{q^{2}}{4}}+\frac{q}{2}}+3 \frac{\int_{0}^{\Theta x} \int_{0}^{x} \chi \frac{C_{1}(\chi, \vartheta)}{P^{3}(\chi, \vartheta)} \frac{\partial C_{1}(\chi, \vartheta)}{\partial \chi} d \chi d \vartheta}{\int_{0}^{\Theta x} \int_{0}^{\mathrm{X}} \chi \frac{\partial C_{1}(\chi, \vartheta)}{\partial \chi} \frac{d \chi d \vartheta}{P^{3}(\chi, \vartheta)}}
\end{aligned}
$$

Here:

$p=\left\{K_{0} \int_{0}^{\Theta} \int_{0}^{\mathrm{x}} \frac{\partial C_{1}(\chi, \vartheta)}{\partial \chi} \frac{\chi}{P^{3}(\chi, \vartheta)} d \chi d \vartheta-3\left[\int_{0}^{\Theta} \int_{0}^{\Theta} \chi \frac{C_{1}(\chi, \vartheta)}{P^{3}(\chi, \vartheta)} \frac{\partial C_{1}(\chi, \vartheta)}{\partial \chi} d \chi d \vartheta\right]^{2}\right\}\left[\int_{0}^{\Theta} \int_{0}^{\Theta} \frac{\partial C_{1}(\chi, \vartheta)}{\partial \chi} \frac{\chi d \chi d \vartheta}{P^{3}(\chi, \vartheta)}\right]^{2}$

where: $\quad K_{0}=3 \varsigma \int_{0}^{\Theta} \int_{0}^{\mathrm{x}} \chi \frac{C_{1}^{2}(\chi, \vartheta)}{P^{3}(\chi, \vartheta)} \frac{\partial C_{1}(\chi, \vartheta)}{\partial \chi} d \chi d \vartheta-\Theta \mathrm{X}$

$$
\begin{aligned}
q & =2\left[\int_{0}^{\Theta} \int_{0}^{\mathrm{x}} \chi \frac{C_{1}(\chi, \vartheta)}{P^{3}(\chi, \vartheta)} \frac{\partial C_{1}(\chi, \vartheta)}{\partial \chi} d \chi d \vartheta\right]^{3}\left[\int_{0}^{\Theta} \int_{0}^{\mathrm{X}} \frac{\partial C_{1}(\chi, \vartheta)}{\partial \chi} \frac{\chi d \chi d \vartheta}{P^{3}(\chi, \vartheta)}\right]^{-3} \\
& -\left[3 \int_{0}^{\Theta} \int_{0}^{\mathrm{x}} \chi \frac{C_{1}^{2}(\chi, \vartheta)}{P^{3}(\chi, \vartheta)} \frac{\partial C_{1}(\chi, \vartheta)}{\partial \chi} d \chi d \vartheta-\frac{\Theta \mathrm{X}}{\varsigma}\right] \int_{0}^{\Theta} \int_{0}^{\Theta \mathrm{x}} \chi \frac{C_{1}(\chi, \vartheta)}{P^{3}(\chi, \vartheta)} \frac{\partial C_{1}(\chi, \vartheta)}{\partial \chi} d \chi d \vartheta\left[\int_{0}^{\Theta} \int_{0} \frac{\partial C_{1}(\chi, \vartheta)}{\partial \chi} \frac{\chi d \chi d \vartheta}{P^{3}(\chi, \vartheta)}\right]^{2}
\end{aligned}
$$

$$
+\left[K_{1}-K_{2}-K_{3}-K_{4}\right]\left[\varsigma \int_{0}^{\Theta} \int_{0}^{\mathrm{x}} \frac{\partial C_{1}(\chi, \vartheta)}{\partial \chi} \frac{\chi d \chi d \vartheta}{P^{3}(\chi, \vartheta)}\right]^{-1}
$$

where:

$$
\begin{aligned}
& K_{1}=\varsigma \int_{0}^{\Theta} \int_{0}^{\Theta} \chi \frac{C_{1}^{3}(\chi, \vartheta)}{P^{3}(\chi, \vartheta)} \frac{\partial C_{1}(\chi, \vartheta)}{\partial \chi} d \chi d \vartheta \\
& K_{3}=\int_{0}^{\Theta} \int_{0}^{\mathrm{X}} C_{1}(\chi, \vartheta) d \chi d \vartheta
\end{aligned}
$$$$
K_{2}=\int_{0}^{\Theta x} \int_{0}^{x} \frac{\partial C_{1}(u, \vartheta)}{\partial \vartheta} d u \frac{\chi d \chi d \vartheta}{\Delta(\chi, \vartheta)}
$$$$
K_{4}=-\mu \int_{0}^{\Theta} \int_{0}^{\mathrm{X}} \int_{0}^{\chi} \frac{\partial C_{1}(u, \vartheta)}{\partial u} d u \frac{\chi d \chi d \vartheta}{\Delta(\chi, \vartheta)}
$$

The second-order approximation of the material concentration gives a possibility to obtain the main physical results of analysis of the spatio-temporal distribution of the considered concentration in different regimes of growth of the considered multilayer structure. If necessary, approximations of the third, fourth, etc. orders could be obtained by analogous way with the second order approximation. In order to verify the obtained results, numerical methods were also used. But numerical methods are less visual than analytical ones.

\section{DISCUSSION}

In this section the spatio-temporal distribution of the concentration of the material of the epitaxial 
layer in the considered heterostructure is analysed. Some examples of calculated spatial distributions of the considered material are presented in figure 2 in comparison with experimental data for different values of temperature of growth and at the same continues of growth. This figure shows an increasing homogeneity of concentration of the material of the epitaxial layer with increasing growth of temperature. At the same time the sharpness of the interface between the layers of the heterostructure decreases.

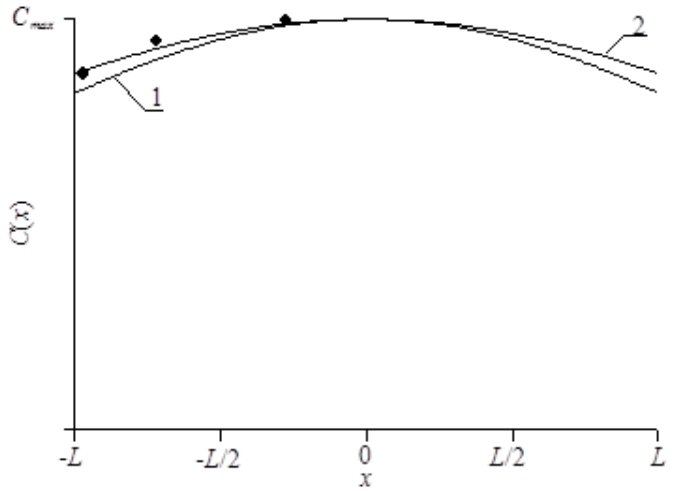

Fig. 2. Distributions of concentration of material of epitaxial layer in considered multilayer structure at different values of temperature of growth. Increasing of number of curves corresponds to increasing of temperature of growth. Points are experimental date, which were obtained by Nazmul et al. (2003).

Figure 3 shows distributions of concentration of material of epitaxial layer in considered multilayer structure at different values of continuance of the growth. Increasing of number of curve corresponds to increaseing of continuance of growth. In this situation again sharpness of the interface be-tween the layers of the heterostructure decreases. At the same time magnitude of the missmutch induced stresses in the heterostructure, caused by the mismatch of the lattice constants of the materials of the epitaxial layer and the substrate, decreases, see for example (Pankratov \& Bulaeva, 2015).

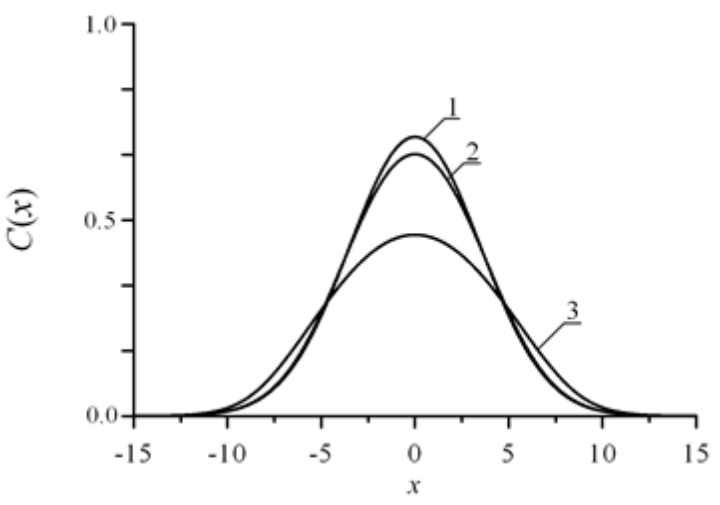

Fig. 3. Distributions of concentration of material of epitaxial layer in considered multilayer structure at different values of continuance of growth. Increasing of number of curves corresponds to increasing of continuance of growth.
Figure 4 shows comparison of normalized dependences of component of displacement vector on coordinate $x$, which is perpendicular to interface between layers of considered multilayer structure, for heated and nonheated multilayer structure. The comparison shows, that heating of the considered multilayer structure leads to decreasing of mismatch induced stresses.

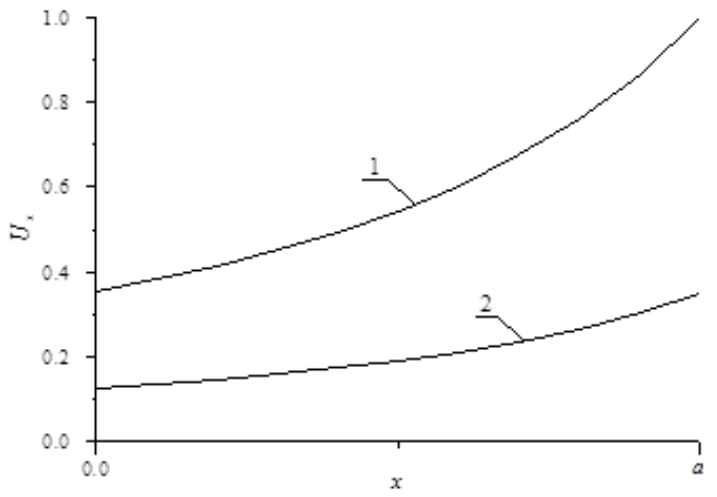

Fig. 4. Normalized dependences of component of displacement vector on coordinate $x$ for nonheated (curve 1) and heated (curve 2) multilayer structure.

\section{CONCLUSIONS}

In this paper an influence of the temperature of growth of epitaxial layers during this technological process was analyzed. Several conditions to increase homogeneity of the considered epitaxial layers were formulated. An analytical approach for analysis of mass and heat transfer was introduced. The approach gives a possibility to take into account changes of parameters of processes simultanciosly in space and in time. At the same time the approach gives a possibility to take into account nonlinearity of the considered processes.

\section{REFERENCES}

Carslaw, H.S., Jaeger, J.C., 1964, Conduction of heat in solids, Oxford University Press, London.

Chakraborty, A., Xing, H., Craven, M.D., Keller, S., Mates, T., Speck, J.S., DenBaars, S.P., Mishrab, U.K., 2004, Nonpolar $\alpha$-plane p-type $\mathrm{GaN}$ and p-n-Junction diodes, Journal of Applied Physics, 96, 4494-4499.

Gotra, Z.Yu., 1991, Technology of microelectronic devices: a Handbook, Radio and communication, Moscow.

Gusev, V.G., Gusev, Yu.M., 1991, Electronics. Higher School, Moscow.

Lachin, V.I., Savelov, N.S., 2001, Electronics. Phenics, Rostovon-Don.

Li, Y., Antonuk, L.E., El-Mohri, Y., Zhao, Q., Du, H., Sawant, A., Wang, Y., 2006, Effects of X-ray irradiation on polycrystalline silicon, thin-film transistors, Journal of Applied Physics, 99, 064501-1-064501-7. 
Lundin, V.V., Sakharov, A.V., Zavarin, E.E., Sinitsin, M.A., Nikolaev, A.E., Mikhailovsky, G.A., Brunkov, P.N., Goncharov, V.V., Ber, B.Ya., Kazantsev, D.Yu., Tsatsul'nikov, A.F., 2009, Effect of carrier gas and doping profile on the surface morphology of movpe grown heavily doped GaN:Mg layers, Semiconductors, 43, 963-967.

Mitsuhara, M., Ogasawara, M., Sugiura, H., 1998, Beryllium doping of InP during metalorganic molecular beam epitaxy using bismethylcyclopentadienyl- beryllium, Journal of Crystal Growth, 183, 38-42.

Nazmul, A.M., Sugahara, S., Tanaka, M., 2003, MBE growth, structural, and transport properties of $\mathrm{Mn} \delta$-doped $\mathrm{GaAs}$ layers, Journal of Crystal Growth, 251, 303-310.

Pankratov, E.L., 2004, Relaxation time of dopant concentration in inhomogenous medium with time varying diffusion coefficient, Applied Nonlinear Dynamics, 12, 35-44.

Pankratov, E.L. Bulaeva, E.A., 2015, Variation of mismatchinduced stress on a heterostructure with changing temperature of growth, Advances in Materials Science and Engineering: An International Journal, 2, 1-10.

Sokolov, Yu D., 1955, About the definition of dynamic forces in the mine lifting, Applied Mechanics, 1, 23-35.

Sorokin, L.M., Veselov, N.V., Shcheglov, M.P., Kalmykov, A.E., Sitnikova, A.A., Feoktistov, N.A., Osipov, A.V., Kukushkin, S.A., 2008, Electron microscopic study of the structure $\mathrm{SiC} / \mathrm{Si}$ (111) obtained by solid-phase epitaxy, Technical Physics Letters, 34, 88-94.

Stepanenko, I.P., 1980, Basis of microelectronics, Soviet radio, Moscow.

Talalaev, R.A., Yakovleva, E.V., Karpova, S.Yu., Makarov, Yu.N., 2001, On low temperature kinetic effects in metalorganic vapor phase epitaxy of III-V compounds, Journal of Crystal Growth, 230, 232-238.

Tikhonov, A.N., Samarskii, A.A., 1972, The mathematical physics equations, Science, Moscow.

Vorob'ev, A.A., Korabl'ev, V.V., Karpov, S.Yu., 2003, Mgdoping $\mathrm{GaN}$ in molecular-beam epitaxy from activated nitride, Semiconductors, 37, 838-842.

\section{PRZEWIDYWANIE ZMIAN WLASNOŚCI EPITAKSJALNYCH WARSTW POPRZEZ DOBÓR POLA TEMPERATURY PODCZAS WZROSTU}

\section{Streszczenie}

W artykule opisano analizę wpływu temperatury na wzrost epitaksjalnych warstw w procesie technologicznym. Sformułowano szereg warunków dla poprawy jednorodności badanych warstw. Wprowadzono analityczne rozwiązanie dla równania transportu masy i ciepła. To rozwiązanie umożliwiło uwzględnienie zmian parametrów procesu zarówno w przestrzeni jak i w czasie. Ponadto przeprowadzone rozwiązanie pozwoliło na uwzględnienie nieliniowości badanego procesu.

Received: March 3, 2020

Received in a revised form: April 1, 2020.

Accepted: April 10, 2020. 